RESEARCH REPORT

\title{
Sex differences in body fat distribution and carotid intima media thickness: cross sectional survey using data from the British regional heart study
}

\author{
Debbie A Lawlor, Shah Ebrahim, Peter Whincup, Jonathan Sterne, Olia Papacosta, Goya \\ Wannamethee, Surinder Dhanjil, Maura Griffin, Andrew N Nicolaides, George Davey Smith
}

J Epidemiol Community Health 2004;58:700-704. doi: 10.1136/jech.2003.014001

See end of article for authors' affiliations

Correspondence to:

Dr D A Lawlor, Department

of Social Medicine,

University of Bristol,

Canynge Hall, Whiteladies

Road, Bristol BS8 2PR, UK;

d.a.lawlor@bristol.ac.uk

Accepted for publication

3 December 2003

\begin{abstract}
Objective: To determine the role of central adiposity in explaining sex differences in carotid intima media thickness (IMT).

Design: Cross sectional survey.

Setting: Two British towns.

Participants: 800 men and women aged $56-75$ years.

Main outcome measures: Carotid IMT.

Results: There was a continuous linear association between waist-hip ratio and IMT in both men and women. The magnitude of the association between waist to hip ratio and IMT was identical in both sexes. In age adjusted analyses IMT was $14 \%$ greater in men compared with women lage adjusted male to female ratio of geometric means $1.14 ; 95 \%$ confidence interval 1.07 to 1.21 ) with adjustment for waist to hip ratio this attenuated to no difference (1.00; 0.92 to 1.09). Adjustment for body mass index and for lifestyle risk factors had very little effect on the sex difference in mean intima media thickness.

Conclusions: Sex differences in body fat distribution may explain sex differences in arterial atherosclerosis.
\end{abstract}

$\mathrm{T}$ he reason for the sex difference in coronary heart disease (CHD) occurrence remains enigmatic. ${ }^{1-6}$ Abdominal obesity is positively associated with CHD risk in both women and men, ${ }^{7-9}$ and it has been suggested that sex differences in fat distribution could explain much of the sex difference in CHD risk. ${ }^{10}{ }^{11}$ In one study the sex difference in CHD risk largely disappeared after adjustment for waist-hip ratio. ${ }^{11}$ The problem with such adjustment is that any risk factor that differs considerably between men and women may "statistically" explain the sex difference in CHD occurrence because of collinearity-in effect it becomes merely a marker for sex. ${ }^{6}$ This dilemma is resolved if two criteria are demonstrated. Firstly, it needs to be demonstrated that the association of abdominal obesity with CHD risk is of similar strength (that is, the regression slope is similar) and is continuous across the two sexes. Secondly, it needs to be demonstrated that the sex difference in CHD risk is attenuated with adjustment for abdominal obesity. ${ }^{12}$ For a dichotomous outcome, such as CHD, large numbers of study participants are required to be able to precisely estimate a similar and continuous association across both sexes (criteria one). ${ }^{11}{ }^{12}$ However, for a continuous outcome, such as carotid artery intima media thickness (IMT), the linear association can be adequately assessed with fewer numbers.

Carotid IMT is a valid indicator of CHD risk. In a prospective study the odds ratio of myocardial infarction for a one standard deviation $(0.163 \mathrm{~mm})$ increase in carotid IMT was 1.43 (1.16 to 1.78 ); when those with a previous infarction or stroke were excluded the association strengthened to 1.51 ( 1.18 to 1.92 ). ${ }^{13}$ Most classic CHD risk factors have been found to have similar effects on IMT in women and men. ${ }^{14-16}$ In cross sectional analysis of the atherosclerosis risk in communities (ARIC) adjustment for waist-hip ratio attenuated but did not completely remove the sex difference in IMT. ${ }^{16}$ Details of the linear association between waist-hip ratio and IMT across the sexes were not presented in that study. The aim of this study was to assess the role of waisthip ratio in explaining sex differences in carotid IMT.

\section{METHODS \\ Subjects}

Data were used from the Dewsbury and Maidstone cohort of the British regional heart study. ${ }^{17}$ The British regional heart study is a prospective study of cardiovascular disease involving 7735 men aged 40-59 years who were randomly selected from the age-sex registers of one general practice in each of 24 British towns and screened between 1978 and 1980. ${ }^{17}$ In 1996, surviving men from two of the British regional heart study towns, Dewsbury in northern England and Maidstone in southern England, were invited for detailed follow up including ultrasonography of carotid and femoral arteries. A random sample of women of similar age to the men (56-75 years) were also selected from each general practice in each of these two towns, and similar invitations to those sent to the surviving cohort men were sent to this new sample of women. ${ }^{18}$ This study is a cross sectional analysis of a group of surviving men from a 20 year old prospective cohort and a group of newly randomly selected women from the same population as the surviving men.

\section{Carotid IMT}

Full details of carotid IMT measurements have been reported. $^{18}$ Measurements were made with an Advanced Technology Laboratories HDI (high definition imaging) 3000 duplex scanner with a high resolution broadband width linear array transducer L 7-4 MHz. All ultrasound measurements were made by two experienced vascular ultrasonographers, and images were recorded on magneto-optical disks and VHS videotapes for further analysis using

Abbreviations: CHD, coronary heart disease; IMT, intima media thickness 
previously described methods. ${ }^{19}$ The within and between ultrasonographer coefficients of variation for IMT measurements were assessed in pilot studies and were between 3\% and $5 \%$. The mean difference between ultrasonographers showed no evidence of bias, and was $-0.005 \mathrm{~mm}$, with $95 \%$ limits of agreement of -0.12 to $+0.11 .^{18}$ In a previous study using data from this cohort it was found that mean IMT at the bulb origin (carotid bifurcation) was more directly associated with clinical CHD than other sites of IMT measurement for both women and men. ${ }^{18}$ This measure of IMT is used as the outcome in our study. Mean IMT of the bifurcation was calculated as the average of the thickest point, including any plaque, of the left and right carotid bulb origin measurements. The bulb origin was defined as the point at which the arterial wall diverges to form the bulb.

\section{Other variables}

Height was measured without shoes using a Harpenden Stadiometer that recorded to the nearest millimetre. Weight was measured in light clothing without shoes using Soenhle portable scales that measured to the nearest $0.1 \mathrm{~kg}$. Waist measurements were taken using the midpoint between the lowest rib and iliac crest and hip measurements using the largest circumference below the waist. A flexible metal tape was used and two measurements taken to the nearest millimetre. A Dinamap 1846SX vital signs monitor was used to measure blood pressure using standard procedures. The Dinamax 1846SX monitor systematically overestimates systolic blood pressure by $8 \mathrm{~mm} \mathrm{Hg}$ and all measurements were corrected for this error. ${ }^{20}$ A sample of non-fasting venous blood was taken for analysis of lipids and glycated haemoglobin.

A self administered questionnaire was used to obtain information on smoking behaviour, alcohol consumption, physical activity, diet, and social class. Subjects were classified into never, former, and current smokers. Alcohol consumption was classified as none, occasional (once or twice a month), weekends only, or daily drinking. Sporting or exercise activity was classified as none, occasional (less than once a month), or frequent (at least once a month). Subjects completed a detailed food item questionnaire and "low fat diet" was defined as mainly using either semi-skimmed, skimmed or no milk and using either low calorie margarine or

\section{Key points}

- The reason for the sex difference in the occurrence of CHD is unclear

- Previous studies have suggested that sex differences in fat distribution may be important, but since the distributions in central adiposity are so different in women and men simple adjustment of the odds ratio of $\mathrm{CHD}$ comparing women with men for waist to hip ratio is uninformative

- It needs to be shown both that there is a continuous linear association across the two sexes in the association between waist to hip ratio and CHD risk, and that adjustment for waist to hip ratio attenuates the association between sex and CHD

- In this study we have shown that there is a linear continuous association between waist to hip ratio and carotid intima media thickness (an indicator of atherosclerosis) across both sexes and in addition that adjustment for waist to hip ratio removes the sex difference in carotid intima media thickness no spread on bread. Social class was derived from the longest held occupation of each man or of each husband in the case of married women and her own occupation in the case of single women. Occupation was coded in accordance with the registrar general's occupational classification (social classes I, II, III non-manual, III manual, IV, V, where I is professional/ managerial and $\mathrm{V}$ unskilled manual labour). ${ }^{21}$

\section{Statistical analysis}

Multiple linear regression was used to assess the effect of adjustment for waist-hip ratio on sex differences in IMT. The outcome in these models was IMT the main predictor variable was sex. Age, waist-hip ratio, body mass index, systolic and diastolic blood pressure, high density lipoprotein cholesterol, triglycerides (logged), and glycated haemoglobin were entered as continuous variables. Smoking, alcohol consumption, exercise, low fat diet, and social class (I, II, III nonmanual, III manual, IV, V) were entered as categorical variables, together with dummy variables representing those for whom data on smoking status $(n=59)$, alcohol $(n=46)$, exercise $(n=56)$, low fat diet $(n=43)$, and social class $(n=55)$ were not available. For continuous variables missing values were not imputed. Sensitivity analyses were undertaken, without these dummy variables, including only subjects with complete data for all categorical as well as continuous variables. The point estimates and $p$ values of these sensitivity analysis were unaltered from those presented here; the confidence intervals were slightly widened with changes at the third decimal place. In all models adjustment was made for age, the clustering effect of each town (to obtain robust standard errors for the calculation of 95\% confidence intervals), and for social class because there was a slight sex difference in the distribution of social class in the cohort.

IMT had a skewed distribution but was log normal and therefore geometric means are presented and logged values were used in the linear regression models; with these transformations residuals in the linear regression models were normally distributed. The resulting regression coefficients of these logged variables were back transformed to provide the ratio of geometric means that were then used to provide the proportionate (expressed as a percentage) difference in IMT level between men and women. All analyses were undertaken using Stata version 7 (Stata Corporation, Texas).

\section{RESULTS}

A total of 375 women and 425 men attended (response rates of $69 \%$ and $83 \%$ respectively) and of these adequate ultrasound images were obtained on 367 women and 418 men. Women who attended and had ultrasound images were younger than non-responders (mean age 65.2 versus 67.7 years, $\mathrm{p}<0.001)$. There was no substantive age difference between responders and non-responders among men (65.8 compared with 65.9 years, $p=0.9)$. The mean $(S D)$ age of female study participants was 65.2 (6.0) and of male study participants was $65.8(5.6), \mathrm{p}=0.18$ for difference between female and male participants.

Figure 1 shows the distribution of waist-hip ratio in women and men; there is little overlap between the sexes. Table 1 shows age adjusted distributions of IMT, waist-hip ratio, and other CHD risk factors in women and men. IMT was thinner in women compared with men (geometric mean $1.36 \mathrm{~mm}$ versus $1.54 \mathrm{~mm}$ ) and women had smaller waist-hip ratios (mean 0.80 versus 0.93 ) than men. Compared with women, men had higher systolic and diastolic blood pressures, higher glycated haemoglobin, and triglyceride levels and lower high density lipoprotein levels and low density lipoprotein levels. The proportion of daily drinkers among men was twice that among women and men were less 


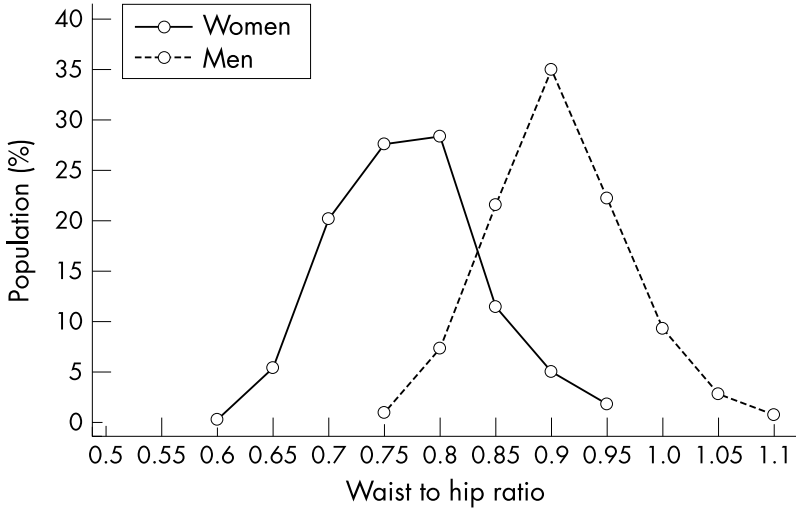

Figure 1 Waist-hip distribution in women and men aged 56-77 from two English towns.

likely to have a low fat diet. There was very little difference between women and men in the proportion undertaking sport or exercise at least monthly. Similar proportions of women and men were current smokers but the proportion of men who were ex-smokers was nearly twice that of women.

Figure 2 shows the unadjusted linear relation between waist-hip ratio and IMT in women and men. This suggests a modest continuous relation across both sexes with similar gradients in the linear relation between waist-hip ratio and IMT in both women and men. After adjustment for age, town, and social class the regression coefficients (95\% confidence intervals) of log IMT on waist-hip ratio were identical in women and men: IMT increased by $8 \%$ (95\% confidence interval: $3 \%$ to $14 \%$ ), $\mathrm{p}<0.001$ for a one standard deviation increase in waist-hip ratio in both sexes.

In both sexes waist-hip ratio was positively associated with triglyceride level, glycated haemoglobin level, and was inversely associated with high density lipoprotein cholesterol (all $\mathrm{p}$ values $<0.01$ ). There was no strong evidence of statistical interaction with sex in the associations between waist-hip ratio and any of the metabolic risk factors (all $\mathrm{p}$ values were equal to or greater than 0.1 ). In women, age was positively associated with waist-hip ratio but in men there appeared to be no association between age and waisthip ratio $(\mathrm{p}$ for interaction $=0.01)$. The age, social class, and town adjusted ratio of male to female IMT geometric means (95\% CI) was 1.14 (1.07 to 1.21$)$ and when further adjustment for waist to hip ratio was made this attenuated

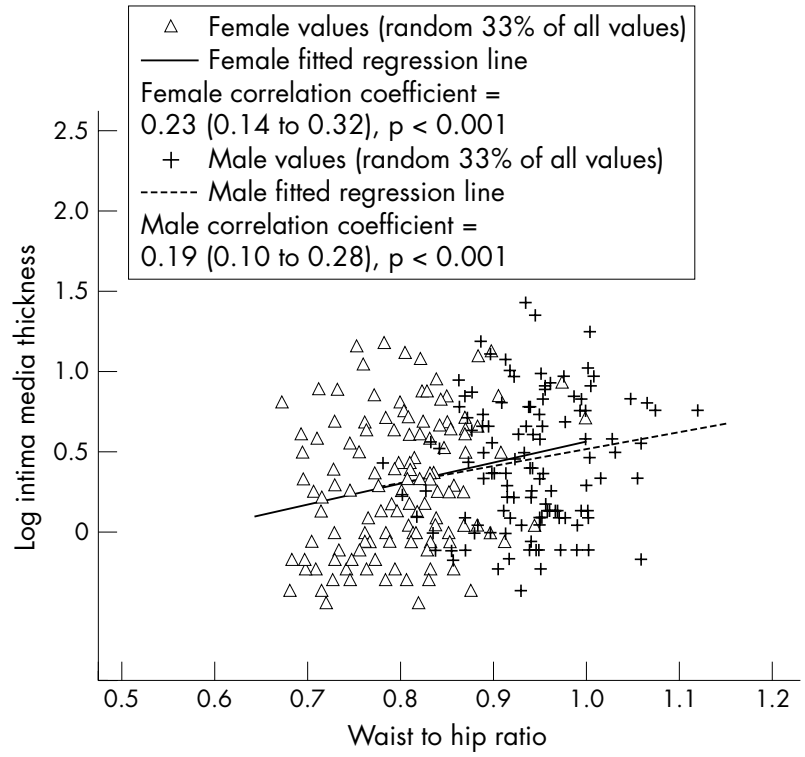

Figure 2 Regression of waist-hip ratio on intima media thickness in women and men aged 56-77 from two English towns.

to 1.00 (0.92 to 1.09$)$. These results have been presented as percentage changes in the first row of table 2-that is, $14 \%$ ( $7 \%$ to $21 \%$ ) and $0 \%(-8 \%$ to $9 \%)$. Adjustment for metabolic risk factors attenuated the association from $15 \%$ to $8 \%$. Adjustment for body mass index and for lifestyle risk factors had very little effect on the sex difference in IMT.

\section{DISCUSSION}

\section{Main findings}

In this study we have shown that the association between waist-hip ratio and carotid IMT is linear and continuous across both sexes and that the magnitude of the association, although modest, is the same in both sexes. Furthermore, adjustment for waist-hip ratio attenuated the sex difference in IMT to the null value.

\section{Study limitations}

We have used carotid IMT rather than CHD as our outcome. Carotid IMT has been shown to be strongly associated with prevalent and incident CHD. ${ }^{13}{ }^{18}$ It has been suggested that the difference in IMT between women and men may be

Table 1 Age adjusted means or prevalences (95\% confidence intervals) of intima media thickness, waist-hip ratio, and risk factors in women and men

\begin{tabular}{|c|c|c|c|}
\hline & Women $(n=367)$ & Men $(n=418)$ & p for difference \\
\hline Intima media thickness* $(\mathrm{mm})$ & $1.36(1.31$ to 1.42$)$ & $1.54(1.49$ to 1.61$)$ & $<0.001$ \\
\hline Waist-hip ratio & $0.80(0.79$ to 0.80$)$ & $0.93(0.92,0.94)$ & $<0.001$ \\
\hline Waist circumference $(\mathrm{cm})$ & $84.90(83.73$ to 86.06$)$ & $97.33(96.25$ to 98.42$)$ & $<0.001$ \\
\hline Hip circumference $(\mathrm{cm})$ & $106.66(105.73$ to 107.58$)$ & 104.41 (103.55 to 105.27$)$ & $<0.001$ \\
\hline Body mass index $\left(\mathrm{kg} / \mathrm{m}^{2}\right)$ & $27.72(27.27$ to 28.16$)$ & $26.91(26.49$ to 27.32$)$ & $<0.001$ \\
\hline $\mathrm{HDLc}(\mathrm{mmol} / \mathrm{l})$ & 1.55 (1.51 to 1.59$)$ & $1.26(1.23$ to 1.29$)$ & $<0.001$ \\
\hline LDLc (mmol/l) & $4.14(4.04$ to 4.24$)$ & 3.65 (3.56 to 3.74$)$ & 0.03 \\
\hline Triglycerides $(\mathrm{mmol} / \mathrm{l})^{*}$ & 1.87 (1.77 to 1.97$)$ & 2.01 (1.92 to 2.12$)$ & 0.005 \\
\hline Glycated haemoglobin (\%) & 4.69 (4.59 to 4.79$)$ & 4.85 (4.75 to 4.95$)$ & 0.01 \\
\hline Systolic blood pressure $(\mathrm{mm} \mathrm{Hg})$ & 144.3 (142.0 to 146.7$)$ & $149.0(146.8$ to 151.2$)$ & $<0.001$ \\
\hline Diastolic blood pressure $(\mathrm{mm} \mathrm{Hg})$ & 77.1 (76.0 to 78.2 ) & 84.3 (83.3 to 85.3$)$ & $<0.001$ \\
\hline Current smoker (\%) & 19.5 (15.5 to 24.3$)$ & 20.3 (16.7 to 24.4$)$ & 0.13 \\
\hline Former smoker (\%) & 28.9 (24.1 to 31.2$)$ & $51.8(46.9$ to 56.5$)$ & $<0.001$ \\
\hline Daily alcohol (\%) & $15.1(11.7$ to 19.4$)$ & 36.4 (31.9 to 41.2 ) & $<0.001$ \\
\hline Exercise at least once a month (\%) & $21.8(17.6$ to 26.6$)$ & $26.2(22.1$ to 30.7$)$ & 0.13 \\
\hline Low fat diet $(\%)$ & 36.1 (31.3 to 41.2$)$ & $27.0(23.0$ to 31.5$)$ & 0.01 \\
\hline Manual social class (\%) & $50.9(45.3$ to 56.4$)$ & 58.6 (53.9 to 63.3$)$ & 0.001 \\
\hline
\end{tabular}


Table 2 Effect of adjustment for central adiposity and other risk factors on the sex difference in carotid intima media thickness. Results show the percentage difference between men and women in carotid IMT $(95 \% \mathrm{Cl})^{*}$

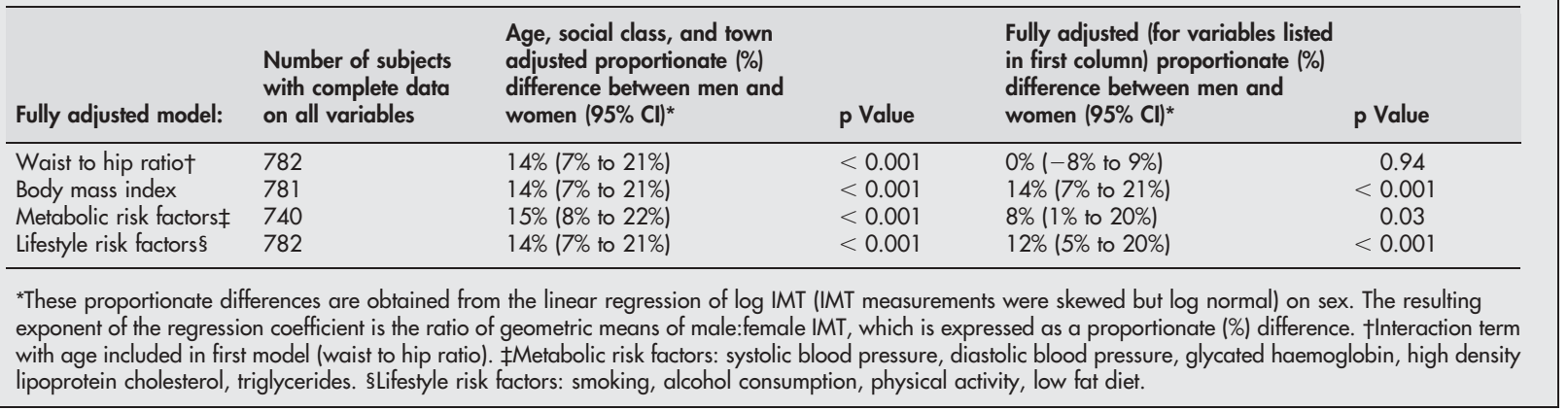

explained by differences in luminal diameter. ${ }^{22}$ However, this has been shown not to be the case in the large Tromso study. ${ }^{14}$ There was a differential in the response between women and men in our study and a difference in the nature of the sampling frame for women and men. Men were survivors of a prospective cohort, whereas women were a newly selected random sample. While among the men responders did not appear to differ from non-responders with respect to age, women who responded were younger than those who did not and women were on average slightly younger than men in the final study sample. All results presented in this study are adjusted for age. Mean blood pressure, lipid levels, and waist-hip ratios for both women and men from this study are similar to those for similar aged women and men from the health survey for England, ${ }^{23}$ and the distributions of IMT for women and men in this study were similar to those of similar aged women and men in other studies. ${ }^{13-15}$ However, the difference in social class distributions between women and men and the lower low density lipoprotein cholesterol level among men compared with women in this study may indicate that there was some selection bias in the study sample. Such selection bias would only importantly bias our results if men with small waist-hip ratios but increased IMT had selectively died during the 20 years follow up of the British regional heart study. Such survivorship bias would be unlikely to affect women who have much lower mortality at these ages. While adjustment for lifestyle factors did not importantly reduce the sex difference in IMT it is possible that our assessment of some of these exposures, for example physical activity and low fat diet, was too crude to allow for full adjustment. Our results are consistent with other prospective studies with CHD outcomes that suggest that sex differences in these life style factors do not explain sex differences in CHD occurrence. ${ }^{56}$ Most (98\%) of the cohort are white. While this means that the results are not necessarily generalisable to other ethnic groups it does mean that the sample is homogenous with respect to ethnicity and therefore not confounded by differences between ethnic groups.

Our findings suggest an important role for abdominal obesity in explaining sex differences in CHD risk and are consistent with an earlier study showing that adjustment for waist-hip ratio removes the sex difference in CHD occurrence. ${ }^{11}$ However, our findings are rather more convincing of such a role than those of the ARIC study, in which adjustment for waist-hip ratio attenuated the sex difference in IMT though did not remove it. ${ }^{16}$ The differences between the two studies may reflect the play of chance, or may be related to the comparatively younger age (45-64 years) of the ARIC population. In addition to waist-hip ratio, diabetes, hyperglycaemia, and other components of the metabolic syndrome seem to account for at least some of the sex difference in CHD risk. ${ }^{24}$ However, in the ARIC study adjustment for hyperglycaemia and diabetes had no effect on the sex difference, ${ }^{16}$ which is surprising.

\section{Implications}

There is a pronounced sex difference in body fat distribution and our results suggest that this has a role in explaining sex differences in arterial atherosclerosis and by implication may be important in explaining some of the sex difference in CHD occurrence. Why are men more prone to central adiposity? It has been suggested that oestrogen protects younger women from CHD via an influence on body fat distribution..$^{25}$ In our study we found that waist-hip ratio increased in women with increasing age but we found no association between age and waist-hip ratio in men. However, all women in our study were post-menopausal and age specific trends show that the decrease in the sex difference in CHD mortality with increasing age is attributable to a deceleration in the male rate rather than an increase in the female rate after the menopause. $^{3}$ The role of male sex hormones in explaining differences in body fat distribution and CHD occurrence is also unclear. ${ }^{\circ}$

Secular trends in CHD mortality suggest that environmental factors are the main drivers of the sex difference in CHD occurrence. ${ }^{2}$ Alcohol consumption, smoking, and physical inactivity have all been found to increase waisthip ratio. ${ }^{26}$ In our study adjustment for these factors had only a small effect on the sex difference in IMT. Within each sex there is considerable variation in waist-hip ratio and while these factors may be important predictors of abdominal obesity, in general they may not fully explain the sex difference in body fat distribution. A recent study of trends in childhood obesity in Britain found that increases in waist circumference among children over the past 30 years greatly exceeded those in body mass index. ${ }^{27}$ Furthermore, it was found that increases in waist circumference among girls over the time period were greater than those seen among boys. $^{27}$ If these trends in childhood central obesity are reflected in similar later adult trends then they could result not only in a slowing in the declines in CHD seen over the past three decades in most industrialised countries, but also a reduction in the sex difference, not because of a positive reduction in men but because of an increase in women.

\section{ACKNOWLEDGEMENTS}

We thank the Stroke Association for funding this study and the Department of Health and the British Heart Foundation for programme grant funding of the British Regional Heart Study. We also thank the CDER Trust for funding the ultrasound equipment. We are grateful to the two general practices that collaborated in the field work, to our field team (Stella Barlow RGN, Annalise Hamilton RGN, Lucy Lennon BSc) who carried out the field work and all the participants of the British regional heart study. 


\section{CONTRIBUTORS}

DAL, SE, and GDS developed the idea for this study and SE, PW, GW, and OP set up and organised data collection for the study. SD and MG undertook the arterial ultrasound scans and ANN interpreted the arterial ultrasound videos. DAL and JS undertook the statistical analysis. DAL wrote the first draft of the paper and all authors have contributed to the final version. DAL and PW will act as guarantor.

\section{Authors' affiliations}

D A Lawlor, S Ebrahim, J Sterne, G Davey Smith, Department of Social Medicine, University of Bristol, Bristol, UK

P Whincup, Department of Public Health Sciences, St George's Hospital Medical School, London, UK

O Papacosta, G Wannamethee, Department of Primary Care and Population Sciences, Royal Free Hospital School of Medicine, London, UK

S Dhaniil, M Griffin, A N Nicolaides, Division of Surgery, Anaesthetics and Intensive Care, Irvine Laboratory, Imperial College School of Medicine, St Mary's Hospital, London, UK

A N Nicolaides, Department of Neurovascular Sciences, The Cyprus Institute of Neurology and Genetics, Nicosia, Cyprus

Funding: this study was funded by the Stroke Association. The Department of Health and British Heart Foundation have provided funding for the British regional heart study. The CDER Trust provided funding for the ultrasound equipment used in this study. DAL was funded by a Medical Research Council/Department of Health (UK) research training fellowship at the time that this work was undertaken and is now funded by a Department of Health (UK) Career Scientist Award. The opinions expressed are those of the authors.

Conflicts of interest: none declared.

\section{REFERENCES}

1 Meilahn E. Sex steroid hormonal influences on coronary artery disease. In: Ness RB, Kuller LH, eds. Health and disease among women. Oxford: Oxford University Press, 1999:155-82.

2 Lawlor DA, Ebrahim S, Davey Smith G. Sex matters: secular and geographical trends in sex differences in coronary heart disease mortality. BMJ 2001;323:541-5.

3 Lawlor DA, Ebrahim S, Davey Smith G. The role of endogenous oestrogen in the aetiology of coronary heart disease: analysis of age related trends in coronary heart disease and breast cancer in England and Wales and Japan. BMJ 2001;325:311-12.

4 Tunstall-Pedoe H. Myth and paradox of coronary risk and the menopause. Lancet 1998:351:1425-7.

5 Isles CG, Hole DJ, Hawthorne VM, et al. Relation between coronary risk and coronary mortality in women of the Renfrew and Paisley survey: comparison with men. Lancet 1992;339:702-6.

6 Barrett-Connor E. Sex differences in coronary heart disease. Why are women so superior? The 1995 Ancel Keys Lecture. Circulation 1997:95:252-64.

7 Lapidus L, Bengtsson C. Regional obesity as a health hazard in women-a prospective study. Acta Med Scand Suppl 1988;723:53-9.
8 Larsson B. Regional obesity as a health hazard in men-prospective studies. Acta Med Scand Suppl 1988;723:45-51.

9 Rexrode KM, Carey VJ, Hennekens $\mathrm{CH}$, et al. Abdominal adiposity and coronary heart disease in women. JAMA 1998;280:1843-8.

10 Wingard DL. Sex differences and coronary heart disease. A case of comparing apples and pears? Circulation 1990;81:1710-12.

11 Larsson B, Bengtsson C, Bjorntop P, et al. Is abdominal body fat distribution a major explanation for the sex difference in the incidence of myocardial infarction? The study of men born in 1913 and the study of women, Goteborg, Sweden. Am J Epidemiol 1992;135:266-73.

12 Lawlor DA, Ebrahim S, Davey Smith G. A lifecourse approach to coronary heart disease and stroke. In: Kuh D, Hardy R, eds. A life course approach to women's health. Oxford: Oxford University Press, 2002:86-120.

13 Bots ML, Hoes AW, Koudstaal PJ, et al. Common carotid intima-media thickness and risk of stroke and myocardial infarction: the Rotterdam Study. Circulation 1997:96:1432-7.

14 Stensland-Bugge E, Bonaa KH, Joakimsen O. Age and sex differences in the relationship between inherited and lifestyle risk factors and subclinical carotid atherosclerosis: the Tromso study. Atherosclerosis 2001;154:437-8.

15 Stensland-Bugge $\mathbf{E}$, Bonaa $\mathrm{KH}$, Joakinsen $\mathrm{O}$, et al. Sex differences in the relationship of risk factors to subclinical athersoslecrosis measured 15 years later. The Tromso study. Stroke 2000;31:574-81.

16 Folsom AR, Eckfeldt JH, Weitzman S, et al. Relation of carotid artery wall thickness to diabetes mellitus, fasting glucose and insulin, body size, and physical activity. Atherosclerosis Risk in Communities (ARIC) Study Investigators. Stroke 1994;25:66-73.

17 Shaper AG, Pocock SJ, Walker $M$, et al. British regional heart study: cardiovascular risk factors in middle-aged men in 24 towns. BMJ 1981;283:179-86.

18 Ebrahim S, Papacosta O, Whincup P, et al. Carotid plaque, intima media thickness, cardiovascular risk factors, and prevalent cardiovascular disease in men and women: the British regional heart study. Stroke 1999;30:841-50.

19 Nicolaides AN, Shifrin EG, Bradbury A, et al. Angiographic and duplex grading of internal carotid stenosis: can we overcome the confusion? $J$ Endovasc Surg 1996;3:158-65.

20 Whincup P, Bruce N, Cook D, et al. The Dinamap 1846SX automated blood pressure recorder: comparison with the Hawksley random zero sphygmomanometer under field conditions. J Epidemiol Community Health 1992;46:164-9.

21 Office of Population Censuses and Surveys and Employment Department Group. Standard occupational classification, volume 1: structure of the classification. London: HMSO, 1990.

22 Bots ML, Hofman A, Grobbee DE. Increased common carotid intima-media thickness: adaptive response or a reflection of atherosclerosis? Findings from the Rotterdam study. Stroke 1997;28:2442-7.

23 Erens B, Primatesta P. Health survey for England 1998: cardiovascular disease. London: The Stationery Office, 1999.

24 Lee WL, Cheung AM, Cape D, et al. Impact of diabetes on coronary artery disease in women and men: a meta- analysis of prospective studies. Diabetes Care 2000;23:962-8.

25 Tremollieres FA, Pouilles JM, Ribot CA. Relative influence of age and menopause on total and regional body composition changes in postmenopausal women. Am J Obstet Gynecol 1996;175:1594-600.

26 Laws A, Terry RB, Barrett-Connor E. Behavioral covariates of waist-to-hip ratio in Rancho Bernardo. Am J Public Health 1990;80:1358-62.

27 McCarthy HD, Ellis S, Cole TJ. Central overweight and obesity in British youth aged 11-16 years: cross sectional surveys of waist circumference. BMJ 2003;326:624-7. 